\title{
ORIGINAL ARTICLE Context-dependent role for chromatin remodeling component PBRM1/BAF180 in clear cell renal cell carcinoma
}

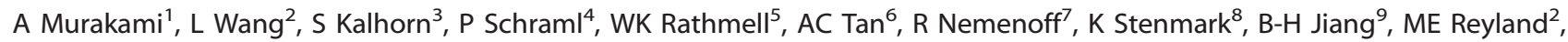 \\ L Heasley ${ }^{2}$ and C-J Hu ${ }^{1,2}$
}

A subset of clear cell renal cell carcinoma (ccRCC) tumors exhibit a HIF1A gene mutation, yielding two ccRCC tumor types, $\mathrm{H} 1 \mathrm{H} 2$ type expressing both HIF1a and HIF2a, and $\mathrm{H} 2$ type expressing HIF2a, but not functional HIF1a protein. However, it is unclear how the $\mathrm{H} 1 \mathrm{H} 2$ type ccRCC tumors escape HIF1's tumor-suppressive activity. The polybromo-1 (PBRM1) gene coding for the BAF180 protein, a component of the SWItch/Sucrose Non-Fermentable (SWI/SNF) chromatin remodeling complex, is inactivated in $40 \%$ ccRCCs, the function and mechanism of BAF180 mutation is unknown. Our previous study indicates that BAF180-containing SWI/SNF chromatin remodeling complex is a co-activator for transcription factor HIF to induce HIF target genes. Thus, our questions are if BAF180 is involved in HIF-mediated hypoxia response and if PBRM1/BAF180 mutation has any association with the HIF1A retention in $\mathrm{H} 1 \mathrm{H} 2$ type cCRCC. We report here that BAF180 is mutated in $\mathrm{H} 1 \mathrm{H} 2$ ccRCC cell lines and tumors, and BAF180 re-expression in $\mathrm{H} 1 \mathrm{H} 2 \mathrm{ccRCC}$ cell lines reduced cell proliferation/survival, indicating that BAF180 has tumor-suppressive role in these cells. However, BAF180 is expressed in HIF1-deficient H2 ccRCC cell lines and tumors, and BAF180 knockdown in H2 type ccRCC cell lines reduced cell proliferation/survival, indicating that BAF180 has tumor-promoting activity in these cells. In addition, our data show that BAF180 functions as co-activator for HIF1- and HIF2-mediated transcriptional response, and BAF180's tumor-suppressive and -promoting activity in ccRCC cell lines depends on co-expression of HIF1 and HIF2, respectively. Thus, our studies reveal that BAF180 function in CCRCC is context dependent, and that mutation of PBRM1/BAF180 serves as an alternative strategy for cCRCC tumors to reduce HIF1 tumor-suppressive activity in $\mathrm{H} 1 \mathrm{H} 2$ ccRCC tumors. Our studies define distinct functional subgroups of ccRCCs based on expression of BAF180, and suggest that BAF180 inhibition may be a novel therapeutic target for patients with $\mathrm{H} 2$, but not $\mathrm{H} 1 \mathrm{H} 2$, ccRCC tumors.

Oncogenesis (2017) 6, e287; doi:10.1038/oncsis.2016.89; published online 16 January 2017

\section{INTRODUCTION}

The incidence of kidney cancers has continued to rise, with 62000 new cases and over 14000 deaths predicted to occur in 2016 in the United States. ${ }^{1}$ Kidney cancer is one of the genitourinary tract cancers that have high mortality rate ${ }^{2-6}$ due to a paucity of effective treatments, indicating an urgent need to better understand the biology of kidney cancer.

The majority of kidney cancers are clear cell renal cell carcinomas (ccRCC). ${ }^{2,4}$ Recent exome sequencing of ccRCC tumors has identified almost universal mutation of the von-Hippel Lindau (VHL) tumor-suppressive gene. ${ }^{7-11} \mathrm{pVHL}$ is a component of the E3 ubiquitin ligase complex, and regulates stability of the transcription factors (TFs), HIF1a and HIF2a. ${ }^{12}$ In cells with wild-type pVHL, HIF1a and HIF2a proteins are targeted for proteasome degradation under normoxic conditions, but stabilized when cells become hypoxic (HX). However, in the absence of a functional pVHL protein, HIF1a and HIF2a are stabilized even under normoxic conditions. HIF1a and HIF2a dimerize with the Aryl Hydrocarbon Receptor Nuclear Translocator (ARNT) to form HIF1a/ARNT (HIF1) or HIF2a/ARNT (HIF2) complexes that induce expression of the genes that regulate metabolism, angiogenesis, cell proliferation and the epithelial-to-mesenchymal transition. $^{13-15}$ Thus, HIF-mediated transcription response is critically important for ccRCC disease.

Despite the significant structural similarities of HIF1a and HIF2a proteins, and their common co-factor, ARNT, HIF1 and HIF2 regulate unique, as well as common target genes. ${ }^{16,17}$ Thus, it is not surprising that both HIF1A and HIF2A gene are required for the ccRCC tumor development/initiation in a mouse model, in which both VHL and TP53 are specifically knocked out in renal tubule epithelial cells. ${ }^{18}$ Despite positive role of both HIF1 and HIF2 in cCRCC initiation, results from clinical and laboratory studies indicate that HIF2 plays a positive role in cCRCC tumor maintenance, ${ }^{19-21}$ whereas HIF1 has a tumor-suppressive role in late stage ccRCC development and in established ccRCC tumors. On the basis of the expression pattern of HIF1a, ccRCC tumors can be divided into two subtypes: $\mathrm{H} 2$ ccRCC tumors that express HIF2a but not a functional HIF1a protein, and $\mathrm{H} 1 \mathrm{H} 2 \mathrm{cCRCC}$ tumors that express both HIF1a and HIF2a protein. ${ }^{2,22}$ Given the evidence that HIF1 functions as a tumor suppressor, an important question that has not been addressed is how $\mathrm{H} 1 \mathrm{H} 2$ ccRCC tumors tolerate HIF1a protein expression.

Exome sequencing has revealed that $40 \%$ of ccRCC tumors also harbor mutations in the polybromo-1 (PBRM1) gene that codes for

\footnotetext{
${ }^{1}$ Molecular Biology Graduate Program, University of Colorado Anschutz Medical Campus, Aurora, CO, USA; ${ }^{2}$ Department of Craniofacial Biology, School of Dental Medicine, University of Colorado Anschutz Medical Campus, Aurora, CO, USA; ${ }^{3}$ Doctor of Dental Surgery Program, School of Dental Medicine, University of Colorado Anschutz Medical Campus, Aurora, CO, USA; ${ }^{4}$ Institute of Surgical Pathology, University Hospital Zurich, Zurich, Switzerland; ${ }^{5}$ Division of Hematology/Oncology, Vanderbilt-Ingram Cancer Center, Vanderbilt University Medical Center, Nashville, TN, USA; ${ }^{6}$ Division of Medical Oncology, Department of Medicine, University of Colorado Anschutz Medical Campus, Aurora, CO, USA; ${ }^{7}$ Division of Renal and

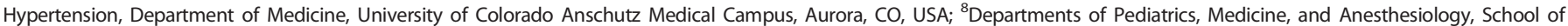
Medicine, University of Colorado Anschutz Medical Campus, Aurora, CO, USA and ${ }^{9}$ Department of Pathology, Anatomy and Cell Biology, Thomas Jefferson University, Philadelphia, PA,

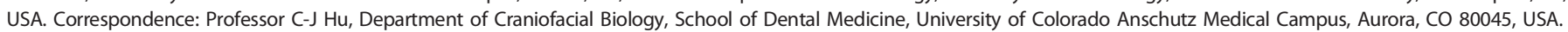
E-mail: Cheng-jun.hu@ucdenver.edu
}

Received 16 August 2016; revised 16 November 2016; accepted 30 November 2016 
the BAF180 protein (we will use the term of BAF180 for the gene and the protein, for simplicity), an accessory component of the PBAF complex, one type of SWI/SNF chromatin remodeling complex. $^{7-11}$ SWI/SNF chromatin remodeling complexes regulate gene transcription through nucleosome disruption and reconstruction in an ATP-dependent manner. Our laboratory has previously demonstrated that the BAF180-containing SWI/SNF chromatin remodeling complex, PBAF complex, acts as a co-activator for HIF1- and HIF2-mediated transcriptional responses by remodeling the promoters of HIF target genes. ${ }^{23}$ These data support a hypothesis that the PBRM1 gene product, BAF180, a unique component of the PBAF complex, may also be important for the HIF-mediated hypoxia response and PBRM1/BAF180 gene mutation may reduce the tumor-suppressive activity of HIF1 in $\mathrm{H} 1 \mathrm{H} 2$ ccRCCs. Thus, the goal of this study was to determine if PBRM1/BAF180 is important for HIF1- and HIF2-mediated transcriptional response, and if the BAF180 gene mutation is associated with HIF1A retention in $\mathrm{H} 1 \mathrm{H} 2$ CCRCC, a tumorsuppressive factor in established $\mathrm{cCRCC}$ tumors. Elucidating the function and molecular mechanism of BAF180 mutation may provide novel therapeutic target for cCRCC patients.

\section{RESULTS}

Mutually exclusive expression of BAF180 and HIF1a protein in $c$ RCC cell lines

HIF1 exhibits tumor-suppressive effects in established ccRCC tumors, ${ }^{24,25}$ but is still expressed in a subset of ccRCC tumors.
Further, the BAF180-containing SWI-SNF chromatin remodeling complex is critical for HIF1-mediated transcriptional response and BAF180 is mutated in a subset of ccRCC tumors. ${ }^{7-11,23}$ These observations prompted us to test the hypothesis that BAF180 mutation is associated with HIF1A retention in $\mathrm{H} 1 \mathrm{H} 2$ CCRCC tumors. Thus, we assessed BAF180, HIF1a and HIF2a protein expression across a panel of ccRCC cell lines (Figure 1a). Consistent with its oncogenic role, HIF2a protein was detected in all ccRCC cell lines under normoxia (Figure 1a). However, HIF1a is lost in KC-12, 769-P, 786-O, RCC10 or truncated in SLR 23 and A498 cells, whereas BAF180 protein expression is lost in RCC4, A704 and SLR25 cell lines (Figure 1a). Interestingly, there is a relationship between BAF180 and HIF1a protein expression, in which cell lines lack BAF180 protein expression (SLR25, A704 and RCC4) expressed full-length HIF1a protein (Figure 1a, indicated by a red arrow), whereas the BAF180-expressing CCRCC cell lines lacked HIF1a protein detection (KC-12, 769-P, 786-O and RCC10) or expressed truncated/non-functional HIF1a proteins (SLR23 and A498; Figure 1a). We next examined HIF1a and BAF180 protein expression in a ccRCC tumor tissue microarray. Twenty-two out of a total of $66 \mathrm{ccRCC}$ tumors exhibited a HIF1a ${ }^{+} / \mathrm{BAF} 180^{-}$pattern, 7 tumors were $\mathrm{HIF} 1 \mathrm{a}^{-} / \mathrm{BAF} 180^{+}, 36$ were double positive and 3 were double negative. Representative images from $\mathrm{HIF} 1 \mathrm{a}^{+} / \mathrm{BAF} 180^{-}$and $\mathrm{HIF} 1 \mathrm{a}^{-} / \mathrm{BAF} 180^{+}$cCRCC tumors are shown (Figure 1b). Thus, expression of full-length HIF1a and BAF180 protein may have a mutually exclusive relationship in most ccRCC cell lines and in some primary tumors.

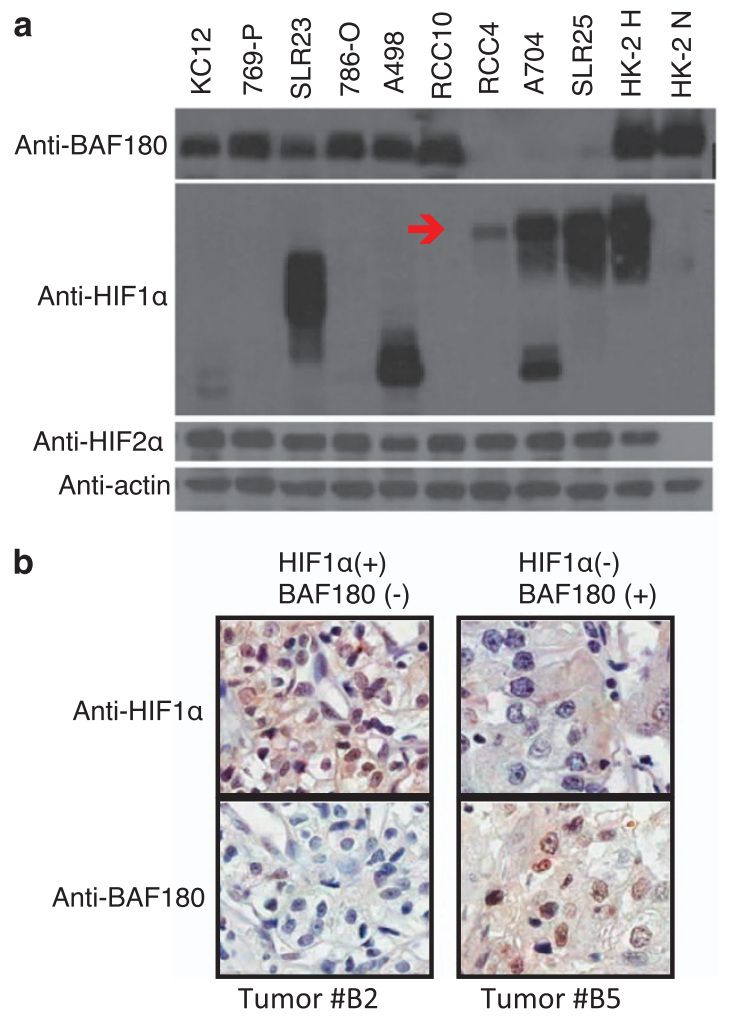

c
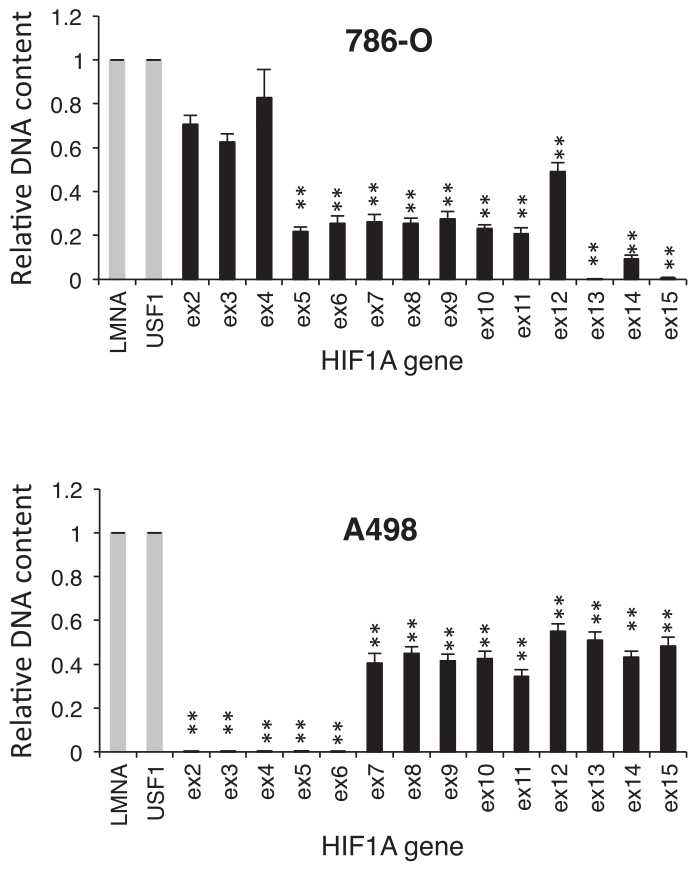

Figure 1. Mutually exclusive expression of full-length BAF180 and HIF1 $\alpha$ proteins in ccRCC cell lines and some primary tumors. (a) Western blot analysis of BAF180, HIF1 $\alpha$ and HIF2 $\alpha$ protein in cCRCC cell lines. The red arrow indicates the position of the full-length HIF1 $\alpha$ protein. A704 cells express full-length as well as truncated HIF1 $\alpha$ protein. Normoxic and hypoxic HK2 cells were used as positive controls for BAF180, HIF1 $\alpha$ and HIF2 $\alpha$ protein detection. (b) Immunohistochemistry staining of HIF1 $\alpha$ or BAF180 proteins in the \#B2 and \#B5 ccRCC tumors in the ccRCC tumor microarray (US Biomax, Derwood, MD, USA, cat. \#BC07014a). (c) qPCR analysis of the abundance of individual exons of the HIF1A gene in genomic DNAs isolated from the indicated cCRCC cell lines. Relative DNA content was normalized to LMNA and USF1 genes as these genes were not amplified or deleted in ccRCC cells. In addition, DNA content of HIF1A exons from HK2 cells were used as calibrators as HIF1A gene is maintained at two copies in HK2 cells. Exons with relative DNA content at 1, 0.5 or close to zero indicate normal, loss of one copy or loss of both alleles in ccRCC cell lines. 
a

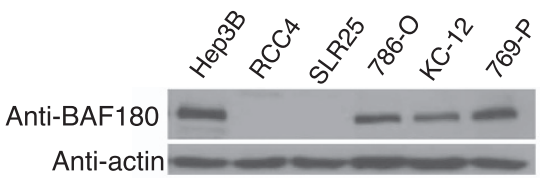

b

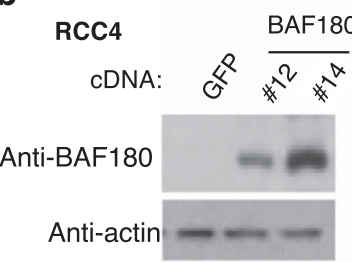

C
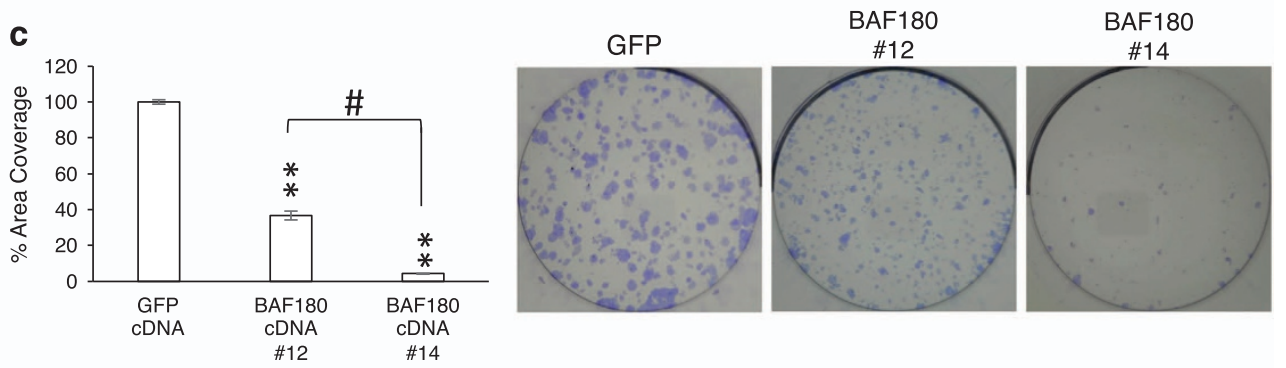

d

e

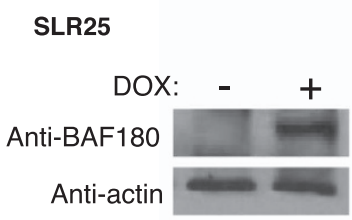

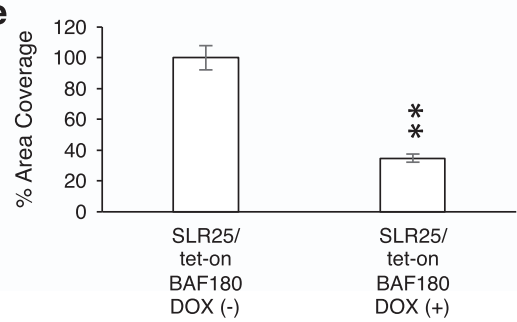

DOX:

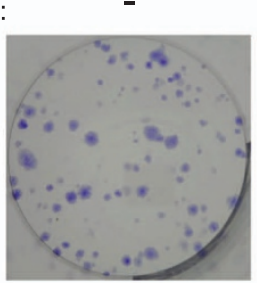

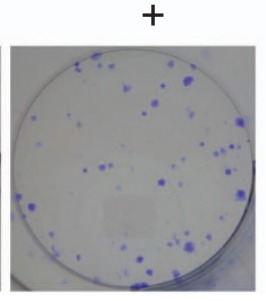

Figure 2. Re-expression of BAF180 in $\mathrm{H} 1 \mathrm{H} 2$ ccRCC lines of RCC4 and SLR25 reduces cell survival/proliferation. (a) Western blot analysis of BAF180 protein in cCRCC cell lines. Beta actin serves as a protein-loading control for this and other WB analysis in the paper. (b) Western blot analysis of BAF180 protein in RCC4 cell clones stably transfected with a BAF180 expression vector or a vector-expressing GFP. (c) Quantification and photos of clonogenic survival assays for RCC4/BAF180 or GFP cells. Quantification was performed using MetaMorph software to measure the total area covered by colonies. (d) Western blot analysis of BAF180 protein expression in SLR25/Tet-on BAF180 cell line with (+) or without $(-)$ doxycycline treatment. (e) Quantification and photos of clonogenic survival assay for SLR25/Tet-on BAF180 cells in the presence (+) or absence $(-)$ of doxcycline.

HIF1A gene mutation is mainly by exon deletion in CCRCC cell lines To determine the molecular mechanism of loss of HIF1a protein expression, or expression of truncated HIF1a protein in ccRCC cell lines, we quantified the abundance of individual exons of the HIF1A gene in genomic DNAs isolated from ccRCC cell lines (Figure 1c and Supplementary Figure 1). Using the non-altered genes of lamin $A / C(L M N A)$ and upstream stimulatory factor 1 (USF1) as DNA-loading controls, and genomic DNA from normal kidney epithelial HK2 cells as a normal DNA copy number control, most HIF1a-defective cCRCC cell lines were found to have loss of one HIF1A allele and loss of several exons of the second allele of the HIF1A gene (Figure $1 \mathrm{c}$ and Supplementary Figure 1). For example, 786-O cells lost exons 5-15 in one HIF1A allele and had a deletion of exons 13-15 in the second allele, also A498 cells lost one HIF1A allele completely and had additional deletion of exons $2-6$ in the second allele. Our data are consistent with previous reports ${ }^{10,11,26,27}$ that $14 q 33.1$ where the HIF1A gene is located, is often associated with copy number loss in ccRCC tumors.

BAF180 has distinct functions in $\mathrm{H} 1 \mathrm{H} 2$ and $\mathrm{H} 2$ ccRCC cell lines To determine the effect of BAF180 protein re-expression in BAF180-deficient cCRCC cell lines, individual RCC4 clones with varying amounts of BAF180 protein re-expression were generated (Figure 2b). A clonogenic assay determined that re-expression of BAF180 decreased the cell survival/proliferation of RCC4 cells in a dose-dependent manner (Figure 2c). Likewise, BAF180 re-expression in a doxycycline-inducible manner also reduced SLR25 cell survival/proliferation (Figures $2 \mathrm{~d}$ and e). Taken together, these results demonstrated that BAF180 re-expression reduces cell survival/proliferation in BAF180-deficient RCC4 and SLR25 cells, consistent with a reported tumor-suppressive role of BAF180 in some ccRCC cell lines. ${ }^{7,28,29}$

To determine if BAF180 also exhibits tumor-suppressive activity in BAF180-expressing ccRCC cell lines (Figure 2a; 786-O, KC-12 and 769-P), we first decreased BAF180 protein levels in 786-O cells using BAF180 short hairpin RNA (shRNA; Figure 3a). Surprisingly, clonogenic assays revealed that $786-O / B A F 180$ shRNA cells exhibited a significant reduction in their clonogenic survival/ proliferation (Figure 3b). BAF180 knockdown in KC-12 and 769-P cells (Figures 3d and f), as well as RCC10 and A498 (not shown) also decreased cell survival/proliferation.

To definitively determine the function of BAF180 in BAF180expressing ccRCC cell lines, we knocked out BAF180 gene expression in 786-O cells using CRISPR-Cas9 technology. Expression of a BAF180 single-guide RNA \#1 (sgRNA\#1) and Cas9 nuclease are expected to introduce double-stranded DNA breaks and subsequently mutation in exon 1 of the BAF180 gene, a location where BAF180 mutations are frequently observed in primary cCRCC tumors ${ }^{7,10,11}$ (Figure $3 \mathrm{~g}$ ). All three CRISPR/Cas9 BAF180-deficient clones were determined to contain one nucleotide deletion, after the expected cleavage site (Figure 3h; sgRNA \#1, clone \#1 and not shown). This mutation led to a premature stop codon within 30 amino acids after the deleted nucleotide and complete loss of BAF180 protein expression (Figure $3 \mathrm{i}$ and not shown). A clonogenic assay showed that 786-O/BAF180 sgRNA \#1 clones \#1, \#2 and \#3 exhibited significantly reduced cell survival/proliferation (Figure $3 \mathrm{j}$ and not shown). To control for potential off-target effects of BAF180 sgRNA $\# 1$, we used another BAF180 sgRNA (sgRNA \#2 in Figure 3g), also located at BAF180 exon 1. Similarly, BAF180 sgRNA \#2 led to the nucleotide deletion (Figure $3 \mathrm{~h}$ and not shown), which resulted in a loss of BAF180 protein expression (Figure $3 i$ and not shown) and a 
reduced cell survival/proliferation (Figure $3 \mathrm{j}$ and not shown). Taken together, these data indicate that BAF180 protein has a tumor-suppressive role in BAF180-deficient cCRCC cells, but an oncogenic role in BAF180-expressing ccRCC cells.
BAF180's tumor-suppressive or -promoting activity depends on expression of HIF1a or HIF2a protein, respectively

To determine whether the loss of tumor-suppressive activity of BAF180 in H2 ccRCC cell lines is due to the loss of HIF1A gene a

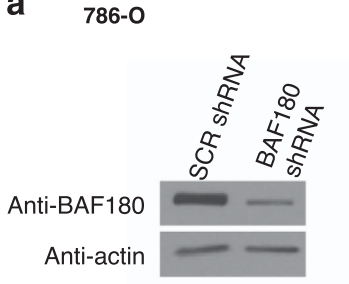

C

KC-12

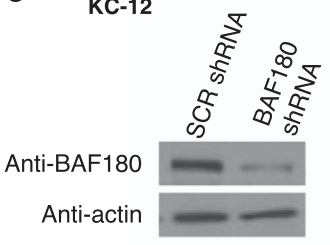

e

769-P

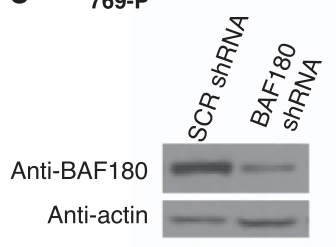

b

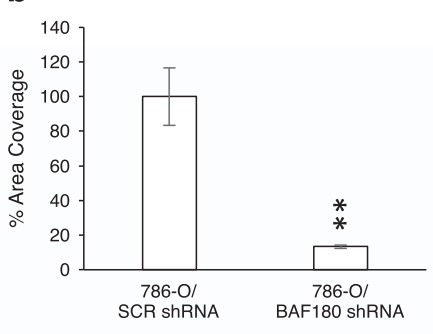

d
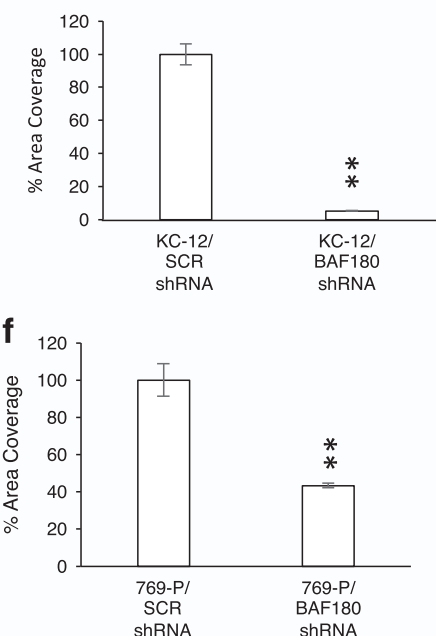

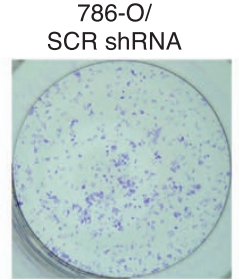

786-O

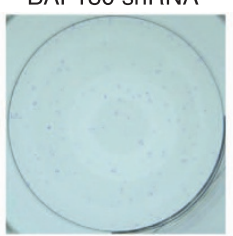

KC-12/ SCR ShRNA

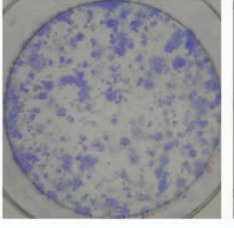

KC-12/ BAF180 shRNA
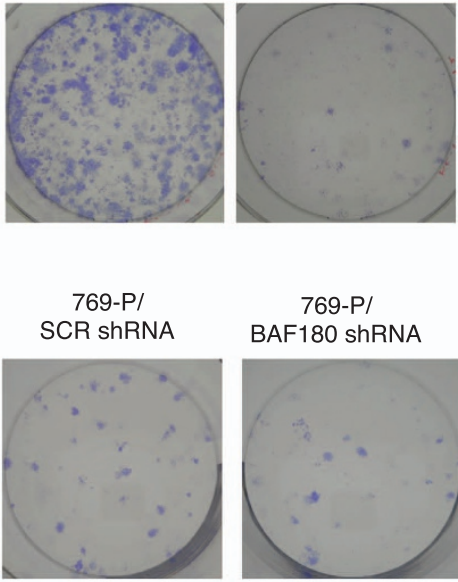

769-P/ BAF180 shRNA

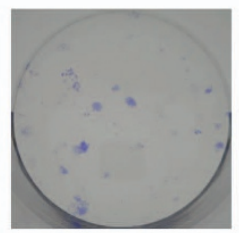

g BAF180 gene sgRNA\#1 sgRNA\#2

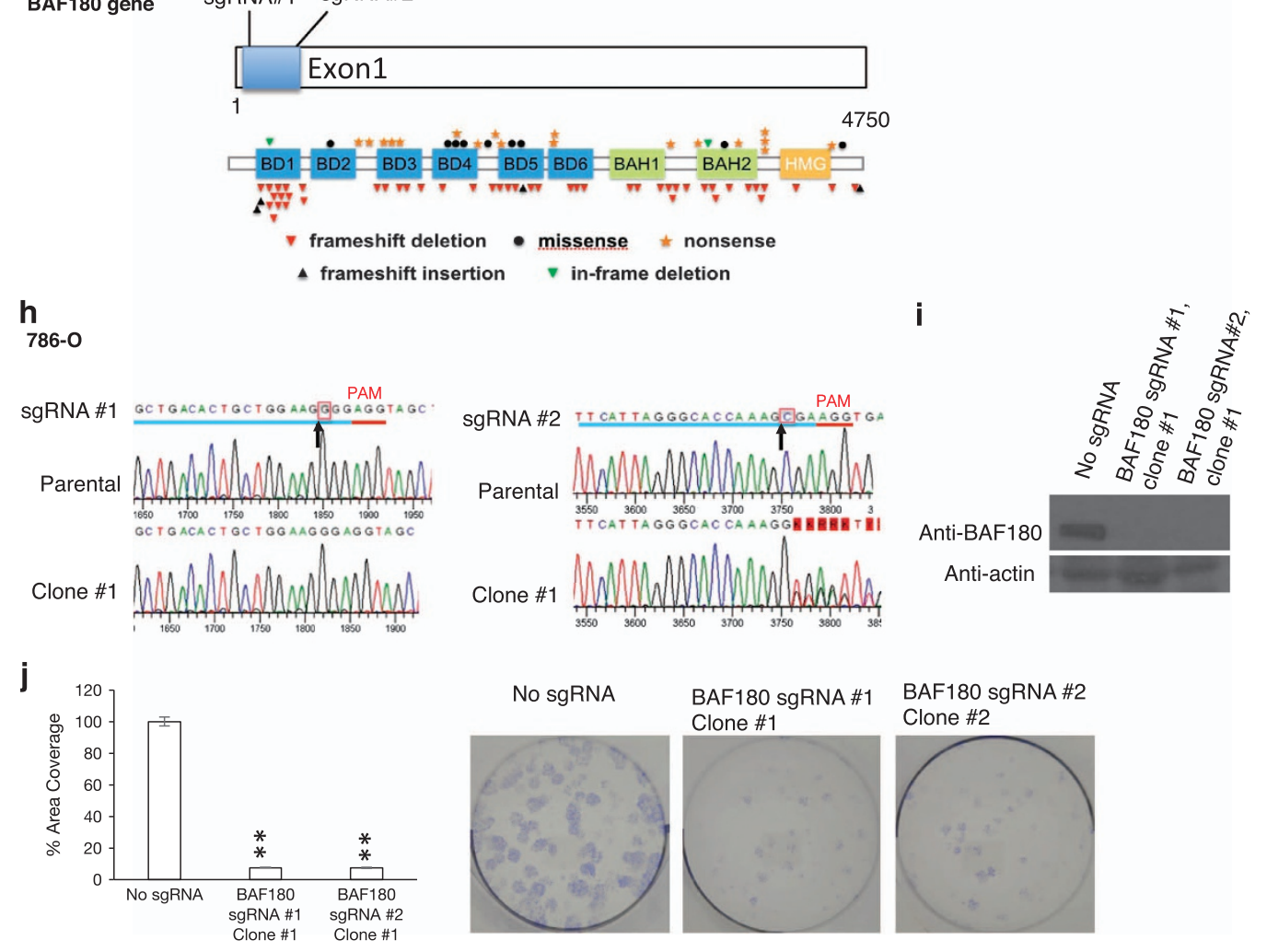

h

786-0

j
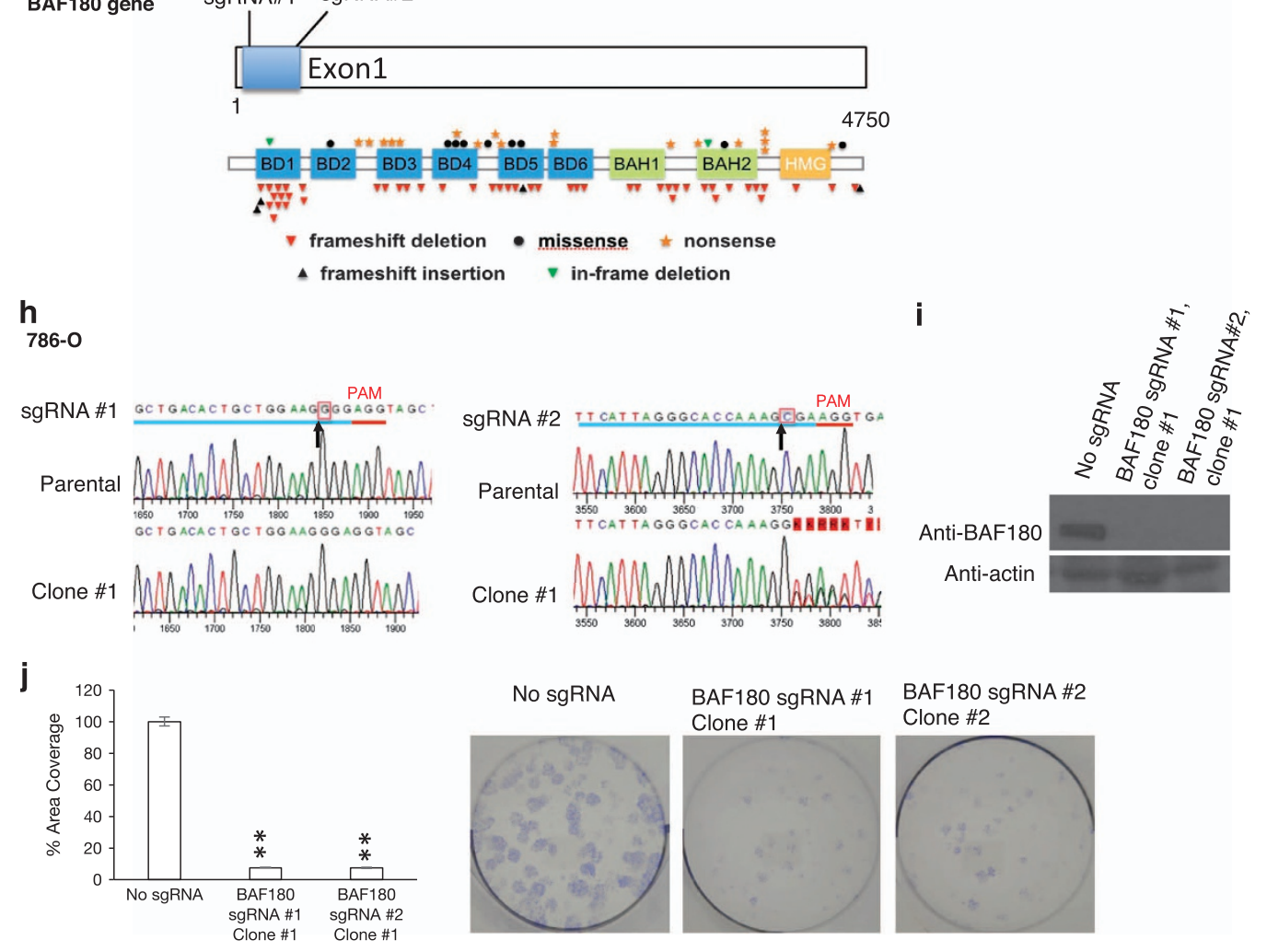

i

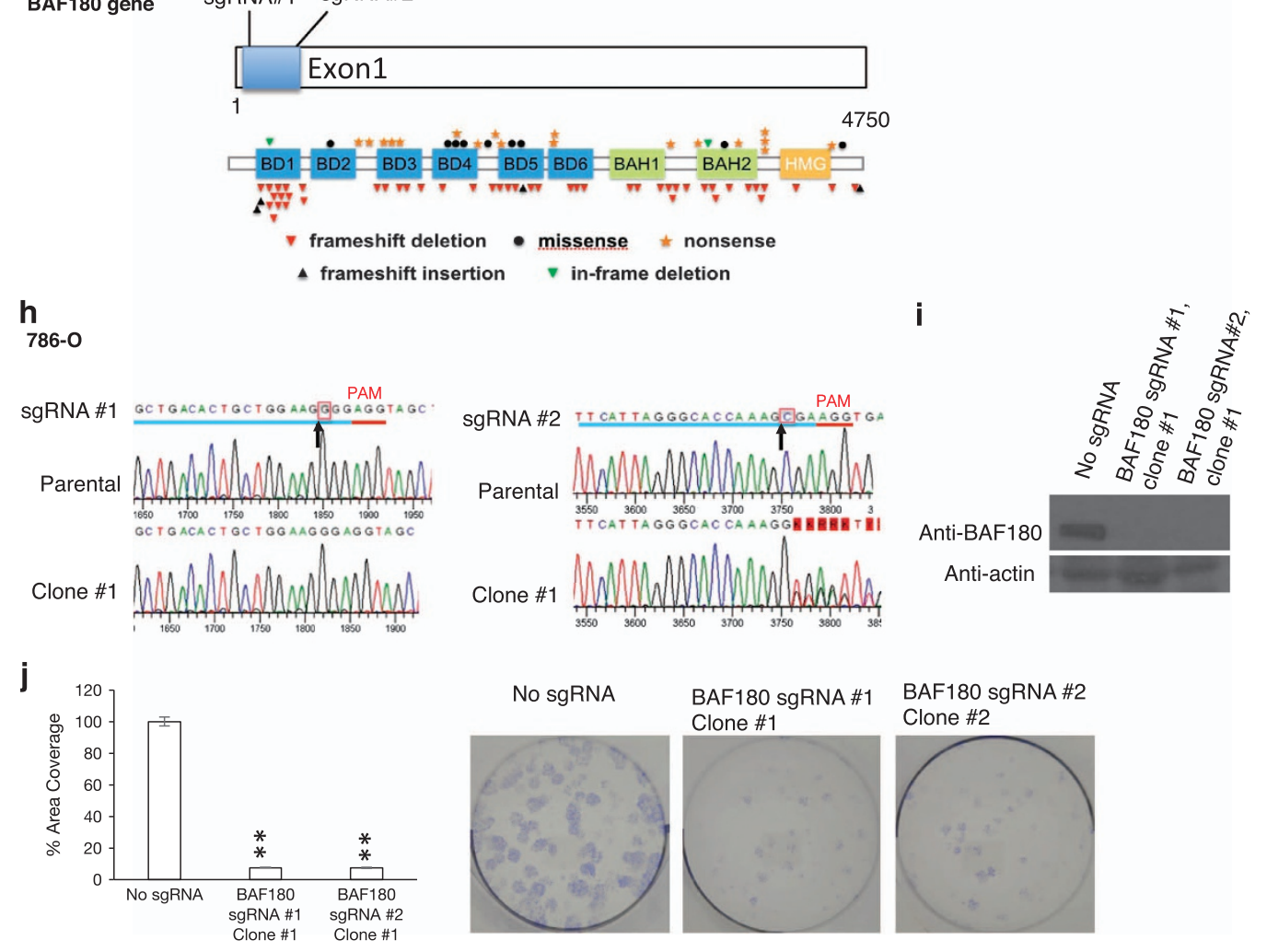


expression, the $\mathrm{H} 2$ ccRCC cell line, 786-O, which normally expresses full-length BAF180, but lacks HIF1a expression, was stably transfected with a mouse HIF1A complementary DNA (cDNA) expression vector (BAF180 WT/HIF1A cDNA; Figure 4a). HIF1a re-expression reduced $786-0$ cell proliferation/survival compared with parental 786-O cells (Figures $4 \mathrm{~b}$ and c), consistent with the reported tumor-suppressive role of HIF1a in cCRCC cell lines. ${ }^{24,25}$ Next, BAF180 was knocked out via CRISPR/Cas9 system to generate a cell line, BAF180 KO/HIF1A cDNA (Figure 4a). BAF180 KO/HIF1A cDNA 786-O cells exhibited increased cell survival/proliferation, compared with BAF180 WT/HIF1A cDNA 786-O cells (Figures $4 \mathrm{~b}$ and $\mathrm{c}$ ), demonstrating that loss of the tumor-suppressive activity of BAF180 in $\mathrm{H} 2$ ccRCC cells is due to the loss of HIF1A gene expression.

$\mathrm{H} 1 \mathrm{H} 2 \mathrm{ccRCC}$ cell lines (RCC4 and SLR25) express both HIF1a and HIF2a, but lack BAF180 protein expression, and re-expression of BAF180 in these cells decreased cell survival/proliferation (Figure 2). To test if BAF180 tumor-suppressive function in $\mathrm{H} 1 \mathrm{H} 2$ cCRCC cell lines depends on HIF1A expression, we generated SLR25/Tet-on BAF180/HIF1A KD cells; to test if BAF180's tumorpromoting activity is associated with HIF2A expression, we also generated SLR25/Tet-on BAF180/HIF2A KD cells, along with SLR25/Tet-on BAF180/SCR shRNA cells as a control (Figure 4d). In the absence of BAF180 expression (no doxycycline), HIF1A KD increased cell survival/proliferation compared with SLR25/SCR shRNA cells. In contrast, HIF2A KD decreased clonogenic survival/proliferation. BAF180 re-expression reduced SLR25/SCR shRNA cell survival/proliferation, which is consistent with data in Figure 2e. Expression of BAF180 in SLR25/HIF2A shRNA cells also reduced cell survival/proliferation compared with the same cells without BAF180 expression, suggesting that BAF180 protein works with the remaining HIF1a to further reduce cell survival (Figures $4 \mathrm{e}$ and f). Furthermore, expression of BAF180 in SLR25/HIF1A shRNA slightly increased clonogenic survival $(P=0.0103)$ compared with the same cells without BAF180 expression, indicating that BAF180 and the remaining HIF2a protein cooperate, at least partially, to increase cell survival (Figures $4 e$ and f). Thus, these data revealed that the function of BAF180 in CCRCC cell lines is dependent on the status of HIF1a or HIF2a expression.

BAF180 inhibition reduces HIF1 and HIF2 target gene expression in Hep3B and ccRCC cells

The ATPase, BRG1, is absolutely required for the function of the PBAF SWI/SNF chromatin remodeling complex; however, the accessory subunits such as BAF180 in the SWI/SNF complex, may or may not be important for a particular process. ${ }^{30-32}$ Although we have reported that the BRG1 knockdown significantly reduces HIF1 and HIF2 to activate their target genes, ${ }^{23}$ the role of BAF180 in HIF1 and HIF2 target gene activation remains unclear. To address this, we used Hep3B cells as a model, as they have a high level of HIF1a and HIF2a expression, and characterization of HIF target gene induction has been well established. ${ }^{16,33-35}$ BAF180
shRNA significantly reduced BAF180 protein levels in normoxic and HX Hep3B/BAF180 shRNA cells (Figure 5a) and BAF180 knockdown markedly reduced the $\mathrm{HX}$ induction of HIF1 target genes (Figure 5b), HIF2 target genes (Figure 5c) and HIF1/HIF2 common target genes (Figure $5 \mathrm{~d}$ ), albeit the levels of reduction were gene specific. We have published that there is a subset of HIF target genes such as GLUT1, LDHA and PGK1, whose HX induction are not affected by BRG1 knockdown. ${ }^{23}$ Consistent with this published study, BAF180 knockdown did not alter the HX induction of these genes (Figure $5 \mathrm{e}$ ).

Knockout of BAF180 in 786-O cells reduced the expression levels of HIF2 target genes (ADM, CST, GLUT1, NDRG1 and ADRP; Figure 5f). Also KC-12/BAF180 shRNA and 769-P/BAF180 shRNA cells exhibited reduced HIF2 target gene expression in comparison with control shRNA (not shown). Conversely, BAF180 re-expression in RCC4 cells increased HIF1 and HIF2 target gene expression in a does-dependent manner (Figure 5g). Thus, BAF180 is important for HIF target gene activation in Hep3B, and in both $\mathrm{H} 2$ and $\mathrm{H} 1 \mathrm{H} 2$ ccRCC cell lines.

BAF180 contributes to chromatin remodeling of the CA9 promoter in HX Hep3B cells

We previously have shown that the PBAF SWI/SNF complex activates transcription of HIF target genes, including CA9 by decreasing nucleosomes associated with the HIF target gene promoter of the CA9 gene. ${ }^{23}$ To test the role of BAF180 in chromatin remodeling of HIF target genes, we performed a qPCR-based nucleosome scanning assays of the CA9 promoter. In Hep3B/SCR shRNA cells, hypoxia treatment significantly reduced nucleosome association on the CA9 promoter, especially in the region from -254 to +113 , which is important for RNA polymerase II and HIF/ARNT binding (the HIF binding site is at -17 ; Figure $6 \mathrm{~b}$ ). HX Hep3B/BRG1 shRNA cells exhibited significant nucleosome association on the CA9 promoter, compared with HX Hep3B/SCR shRNA cells. Hep3B/BAF180 shRNA cells exhibited intermediate levels of nucleosome association on the CA9 promoter, compared with nucleosome association in $\mathrm{HX} \mathrm{Hep} 3 \mathrm{~B} / \mathrm{SCR}$ shRNA and Hep3B/BRG1 shRNA cells (Figure 6b). Thus, our findings reveal that BAF180 contributes significantly to chromatin remodeling of the CA9 promoter in HX Hep3B cells.

\section{DISCUSSION}

HIF1 displays tumor-repressive activity in established ccRCC tumors. Recently, it has been shown that a subset of cCRCC tumors still retain HIF1A gene expression; ${ }^{24,36}$ however, it is unclear how these HIF1a-expressing cCRCC tumors tolerate HIF1 tumor-suppressive activity. Likewise, the BAF180 gene is mutated in $40 \%$ cCRCC tumors, but the reason for BAF180 mutations in these BAF180-deficient ccRCC tumors is not known. Furthermore, a subset of cCRCC tumors retain BAF180 gene expression, the function of BAF180 in these BAF180-expressing cCRCC cells is also

Figure 3. BAF180 knockdown in BAF180-expressing H2 ccRCC cell lines reduces cell survival/proliferation. (a, c, e) Western blot analyses of BAF180 protein in 786-O (a), KC-12 (c) and 769-P (e) H2 cCRCC cells targeted with non-targeting scramble (SCR) shRNA or BAF180 shRNA. (b, d, f) Quantification and photos of the clonogenic survival assay for 786-O (b), KC-12 (d) and 769-P (f) cells targeted with control scr-shRNA or BAF180 shRNA. (g) The location of BAF180 sgRNA \#1 and \#2 relative to the BAF180-coding region are shown (upper). The diagram (lower) shows the type and frequency of mutations in the BAF180 gene, found in ccRCC tumors, adapted from the article by Valera et al. ${ }^{7}$ (h) DNA sequence and sequencing profiles of parental BAF180 DNA (parental) and BAF180 DNA after targeted by BAF180 sgRNA \#1 (left) and \#2 (right). The blue lines indicate the target sequences of sgRNAs and the red lines showing the protospacer adjacent motif sequence. The black arrows indicate the positions that double-strand DNA cleavages are expected to occur by the sgRNA-led Cas 9 enzyme. The red boxes are to indicate the nucleotides that have been deleted by CRISPR-Cas9 system. SgRNA \#2 Clone \#1 shows a mixed sequence after the cleavage site, probably due to the fact that the deletion in the BAF180 allele 1 is different from the deletion in BAF180 allele 2. (i) Western blot analysis of BAF180 protein in 786-O/Cas9 cells expressing no sgRNA, or PBRM1 sgRNA \#1 (clone \#1) or \#2 (clone \#1). (j) Quantification and photos of the clonogenic survival assay for 786-O/Tet-on Cas9 clones without sgRNA expression, or with expression of BAF180 sgRNA \#1 (clone \#1) or \#2 (clone \#1). 
a

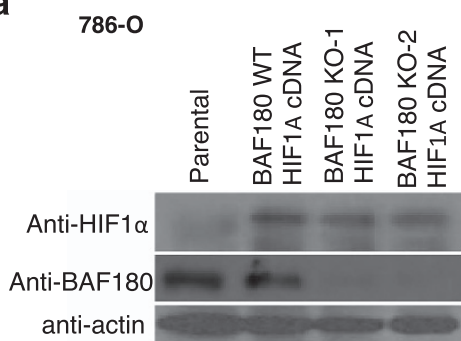

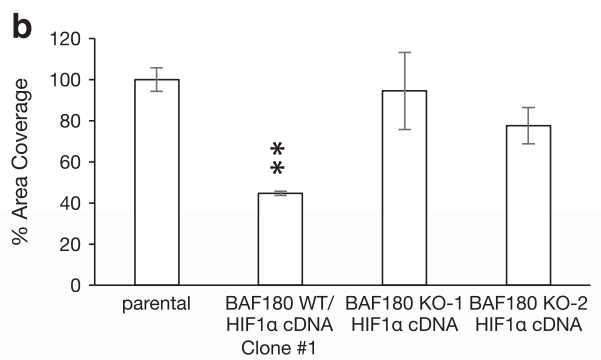

C

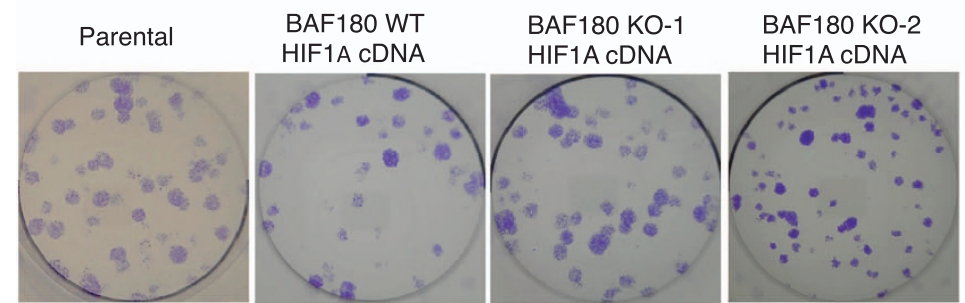

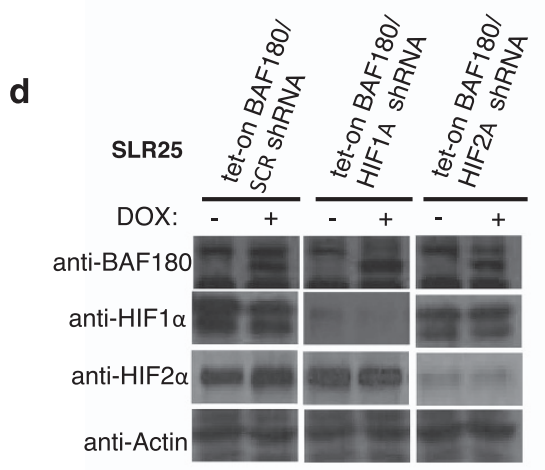

f

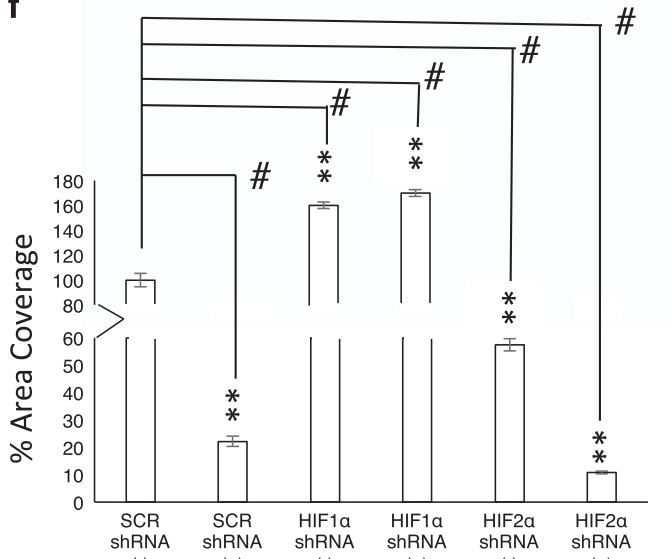

e

DOX: $\quad-\frac{1}{2}+$

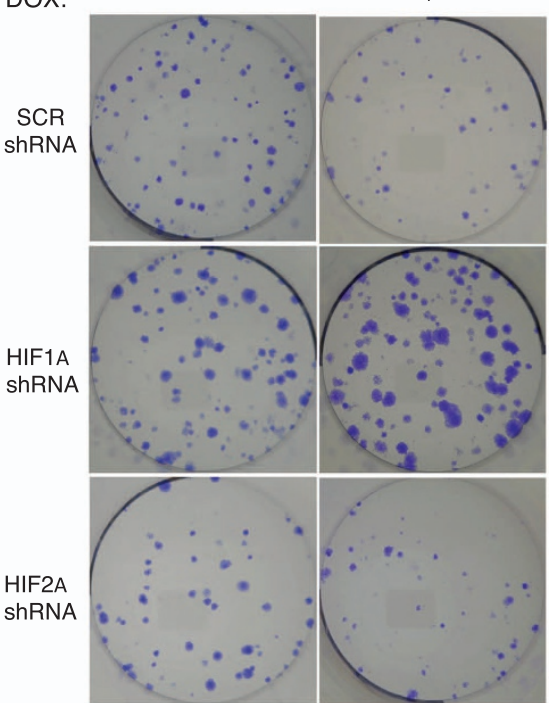

Figure 4. BAF180's tumor-suppressive or -promoting activity in cCRCC cell lines is HIF1 $\alpha$ or HIF2 $\alpha$ dependent. (a) Western blot analysis of HIF1 $\alpha$ and BAF180 protein in parental 786-0 (parental), 786-0 with HIF1 $\alpha$ re-expression (BAF180 WT/HIF1A cDNA) and 786-0 with HIF1 $\alpha$ re-expression, but PBRM1 knockout by sgRNA \#1, clone \#1 and clone \#2 (BAF180 KO-1 or -2/HIF1A cDNA). (b, c) Quantification (b) and photos (c) of clonogenic survival assay in the indicated 786-O cells. (d) Western blot analysis of BAF180, HIF1 $\alpha$ and HIF2 $\alpha$ proteins in SLR25/Tet-on BAF180 cells stably targeted with control, HIF1A or HIF2A shRNA in the absence of $(-)$ or presence of doxycycline (+). (e, f) Quantification (e) and photos (f) of clonogenic survival assay of the SLR25/Tet-on BAF180 cells stably targeted with control, HIF1A or HIF2A shRNA in the absence of $(-)$ or presence of doxycycline $(+)$. All the samples are normalized to SCR non-targeting shRNA without doxycycline treatment.

unknown. In this report, we determined that BAF180 gene mutation, and the subsequent lack of BAF180 protein expression, is observed only in ccRCC cell lines that maintain full-length HIF1a protein expression. Re-expression of the BAF180 protein in these BAF180-deficient H1H2 ccRCC cells increases HIF1 target gene expression and inhibits cell proliferation/survival. Thus, our data indicate that mutation of BAF180 is an alternative strategy for CCRCCs to reduce the tumor-suppressive activity of HIF1. However, BAF180 is expressed in $\mathrm{H} 2$ cCRCC cell lines in which the HIF1A gene is mutated. Reduction or knockout of BAF180 gene in these BAF180-expressing ccRCC cells reduces HIF2 target gene expression and cell proliferation/survival, indicating that BAF180 

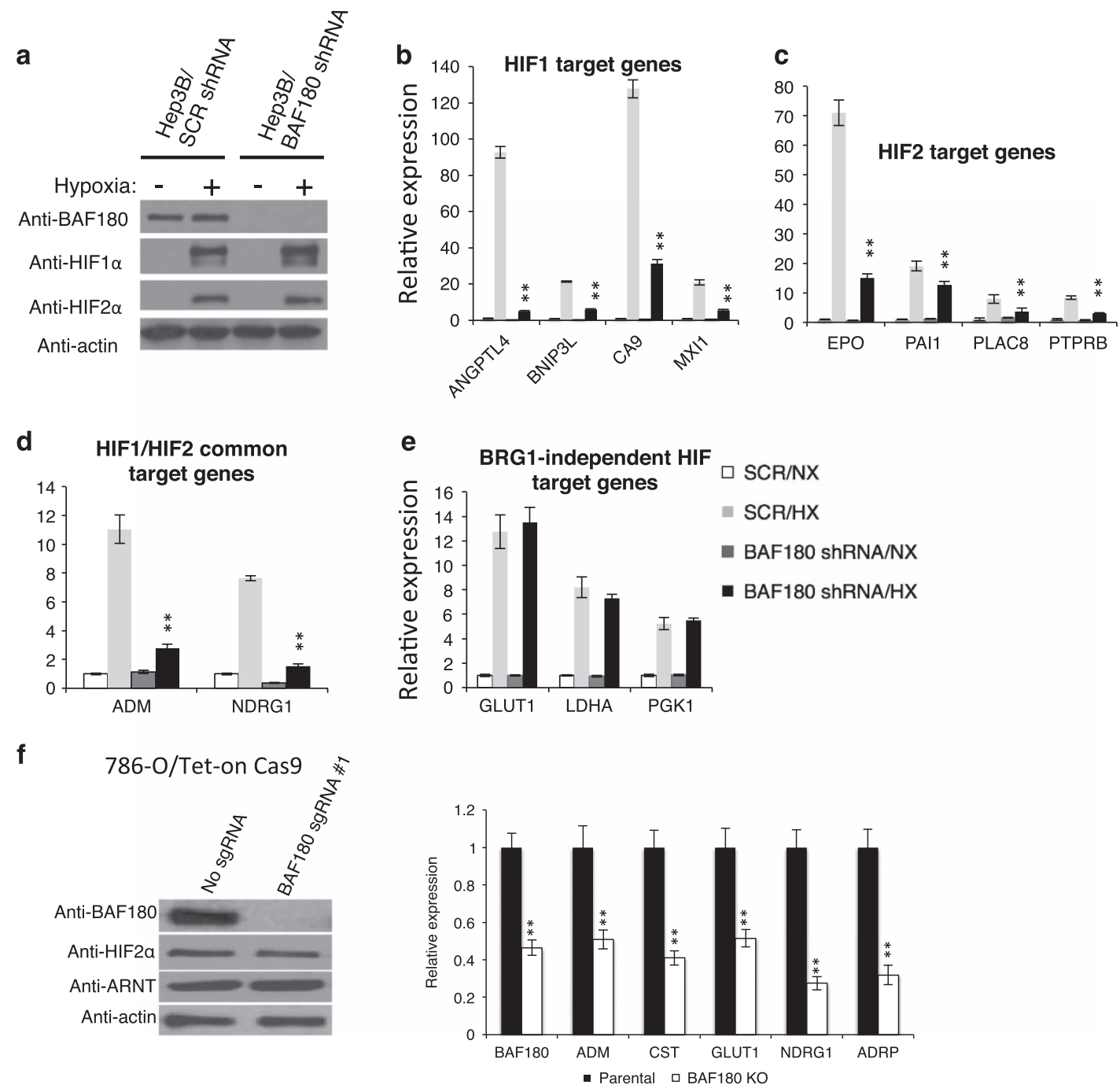

g RCC4/GFP or BAF180 CDNA
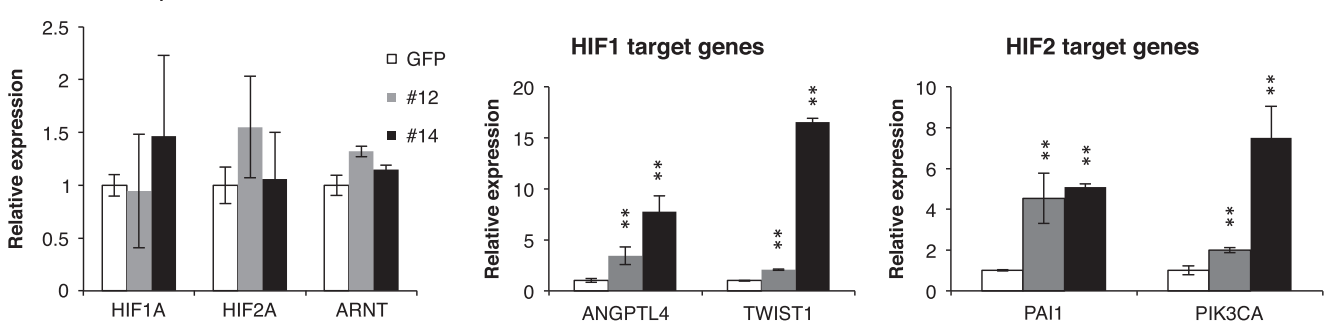

Figure 5. BAF180 is required for strong expression of HIF1 and HIF2 target genes in hypoxic (HX) Hep3B cells and pVHL-deficient ccRCC cell lines. (a) Western blot analysis of BAF180, HIF1 $\alpha$ and HIF2 $\alpha$ proteins in normoxic (NX) and HX Hep3B cells stably transduced with non-targeting control (SCR shRNA) or BAF180 shRNA. (b-e) qRT-PCR analysis of messenger RNA (mRNA) levels of HIF1 target genes (b), HIF2 target genes (c), HIF1/HIF2 common target genes (d) and BRG1-independent HIF target genes (e) in NX and HX Hep3B cells stably targeted with control or BAF180 shRNA. (f) qRT-PCR analysis of mRNA levels of known HIF2 target genes in 786-O/Tet-on Cas9 cells with no sgRNA or BAF180 sgRNA \#1 (right). Expression levels of BAF180, HIF2 $\alpha$ and ARNT protein in these cells were also shown (Left). (g) qRT-PCR analysis of mRNA levels of known HIF1 and HIF2 target genes, as well as HIF and ARNT in RCC4 cells stably expressing GFP (control) or BAF180 protein. Clones \#12 and \#14 express low and high BAF180 protein levels, respectively.

has oncogenic activity in this setting. Thus, our data indicate that $B A F 180$ is not a bona fide tumor-suppressor or oncogene, and BAF180's function in ccRCC is context dependent. These findings are very novel, as the current hypothesis accepted by most investigators is that BAF180 is a bona fide tumor suppressor and loss of BAF180 promotes cancer by re-targeting the SWI/SNF complex to a set of cancer-promoting genes that are not normally regulated by the SWI/SNF complex. ${ }^{37}$
Mutations in the BAF180 gene or downregulation of BAF180 gene expression has been observed in various cancer types, including $\mathrm{CCRCC}$, suggesting a tumor-suppressor function. 7,10,11,38-42 Our data confirmed that BAF180 is mutated in a subset of ccRCC cell lines (Figure 1a and Supplementary Figure 2a) and primary tumors (Figure $1 \mathrm{~b}$ and not shown). Re-expression of PBRM1 in BAF180-deficient ccRCC cell lines, both in stable or inducible manner (Figure 2), reduce ccRCC proliferation/survival, further 
a

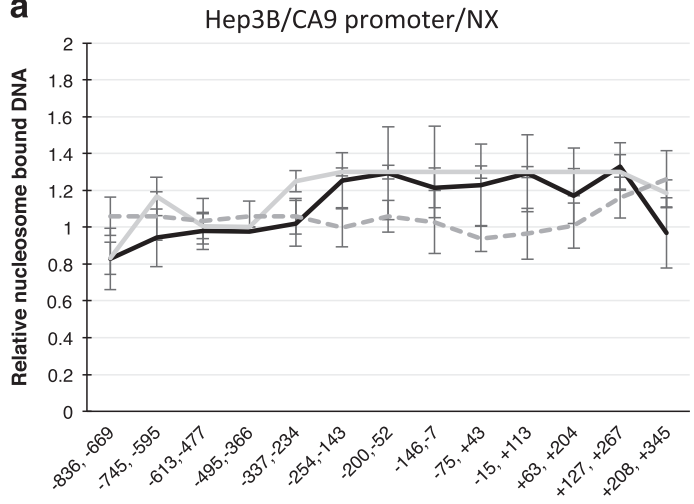

b

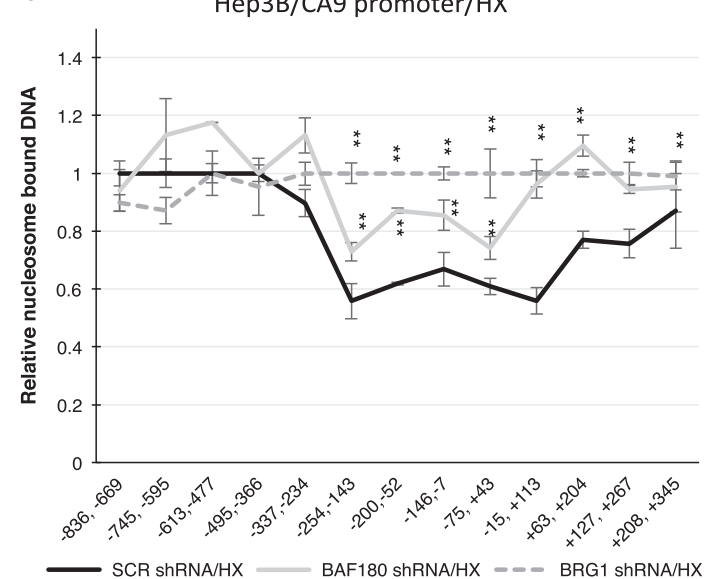

Figure 6. BAF180 expression is important for efficient de-association of nucleosome on the CA9 promoter in hypoxic (HX) Hep3B cells. (a, b) Nucleosome scanning assay of the CA9 promoter in Hep3B cells stably transduced with non-targeting SCR, or BRG1, or BAF180 shRNA under normoxia (a) or hypoxia (b). The location of the primer sets are shown relative to the transcription start site (+1). A region spanning -713 to -477 was used as an internal DNA-loading control, as the readouts in this region did not differ between normoxic (NX) and HX, nor between cells with WT BRG1 and BRG1 knockdown. Relative fractions of nucleosome DNA indicate the levels of nucleosome-bound DNA relative to those for an internal control.

confirming that BAF180 is a tumor-suppressive protein in the BAF180-deficient ccRCC cell lines, which is consistent with published papers. ${ }^{7,28,29}$

Although the previous studies $7,28,29$ did not address the molecular mechanism for BAF180 mutation in CCRCC, we have shown here for the first time that BAF180 mutation is to reduce HIF1's tumor suppressive in cCRCC. This conclusion is first supported by our novel observation that BAF180 and HIF1A gene mutations are mutually exclusive in ccRCC cell lines (Figure $1 \mathrm{a}$ and Supplementary Figure 2a). We consider ccRCC cell lines expressing truncated HIF1a proteins as a HIF1a-deficient cell line, as a previous report indicates that the truncated HIF1a protein is not functional. ${ }^{26}$ By searching the literature, we have found additional cCRCC cell lines that exhibit mutually exclusive expression of BAF180 and HIF1a proteins, although these publications were focusing on BAF180 expression and made no connection between HIF1A mutation and BAF180 mutation. ${ }^{8,43}$ For example, H2 ccRCCs (SKRC-17, SKRC-59 and UMRC-6) express BAF180 protein, whereas $\mathrm{H} 1 \mathrm{H} 2$ ccRCCs cell lines (CAKI-2 and UMRC-2) lack BAF180 expression. ${ }^{43}$ Further, $\mathrm{H} 1 \mathrm{H} 2$ cCRCC cell lines RCC ER, RCC FG2 and RCC MF contain BAF180 gene mutations. ${ }^{8}$ We also found two outliers (Supplementary Figure 2). SLR24 cells are double negative, whereas SLR22 cells are double positive, although both HIF1a and BAF180 protein are significantly reduced in SLR22 cells. We speculate that these outliers are formed in cells with initial BAF180 reduction (SLR22) or mutation (SLR24), followed by further HIF1A reduction (SLR22) or HIF1A mutation (SLR24).

Our experimental data (Figure 1a and Supplementary Figure 2) and our literature analysis above have established the mutually exclusive relationship of BAF180 and HIF1a protein expression in the majority $(19 / 21=90 \%)$ of ccRCC cell lines, our own data (Figure $1 \mathrm{~b}$ ) and analysis of the published data sets (see below) also support such relationship in a subset of the primary ccRCC tumors. For example, by analysis of HIF1A copy number and exome sequencing, ${ }^{10}$ TCGA found that four CCRCC tumors are HIF1 activity deficient, due to loss of one HIF1A allele and have nonsense mutations in the second allele of HIF1A gene. Interestingly, we found that these HIF1A mutant cCRCC tumors express WT BAF180 gene. ${ }^{10}$ Also Sato et al. ${ }^{11}$ reported 17 HIF1a-negative CCRCC tumors and we found that 13 out of the 17 (76\%) HIF1a-negative ccRCC tumors express BAF180 proteins (one-tailed Fisher's exact test: $P=0.0245$ ). Consistent with these data, our own immunohistochemistry staining of BAF180 and
HIF1a in CCRCC tumor tissues (BC0714a, US BioMax) found 7

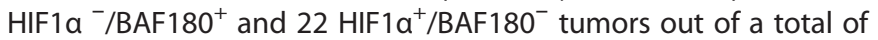
66 ccRCC tumors.

The conclusion that BAF180 protein's tumor-suppressive activity in CCRCC is HIF1 dependent is also supported by functional studies, in which expression of HIF1a in $\mathrm{H} 2$ 786-O cells switches BAF180 from a oncogenic protein to tumor-suppressive protein (Figures $4 \mathrm{~b}$ and $\mathrm{c}$ ), although knockdown of $\mathrm{HIF} 1 \mathrm{a}$ in $\mathrm{H} 1 \mathrm{H} 2$ SLR25 cells turns BAF180 from a tumor-suppressive protein to a tumor-promoting protein (Figures $4 \mathrm{e}$ and $\mathrm{f}$ ).

Our data strongly support a notion that BAF180's activity in CCRCC is closely linked with the TFs HIF1 and HIF2. However, this conclusion does not exclude the possibility that BAF180-containing SWI/SNF chromatin remodeling complex could also potentially work with other TFs and regulate other genes/pathways under different conditions. In fact, multiple publications reported that BAF180 is required for $\mathrm{p} 53$ to activate p21 expression under condition of p53 activation. ${ }^{29,44,45}$ Although p53 can be activated in $\mathrm{ccRCC}^{29}$ it is unlikely that p53 is activated in ccRCC cells under our study condition that cells are cultured under normoxia. In contrast, HIF is constitutively active in cCRCC cell lines under normoxia (Figure 1a). This might explain why the function of BAF180 in cCRCC is closely associated with HIF, not or less with other TFs such as p53.

In conclusion, we show, for the first time, that HIF1A and BAF180 protein expression are mutually exclusive in $\mathrm{ccRCC}$ cell lines and some primary tumors. Further, our results indicate that BAF180's function in cCRCC is closely associated with HIF1 or HIF2 activity, the dominant TFs/pathway in the ccRCC tumors. These findings are of significance for personalized medicine, in which the inhibition of the BAF180 activity may be beneficial for H2 cCRCC patients, but ineffective for $\mathrm{H} 1 \mathrm{H} 2$ ccRCC patients.

\section{MATERIALS AND METHODS}

\section{Cell culture}

HK2 cells were cultured in Hyclone (Logan, UT, USA) Dulbecco's modified Eagle's Medium (DME) and Ham's F-12 nutrient mixture 1:1 $(1 \times)$ supplemented with $10 \%$ fetal bovine serum (FBS), $2.5 \mathrm{~mm}$ L-glutamine, $15 \mathrm{~mm}$ HEPES buffer and $1 \times$ insulin-transferrin-selenium (ITS). 786-O, A498, RCC10, RCC4 and A704 cell lines were cultured in DMEM (Mediatech, Inc., VA, USA) supplemented with $10 \%$ FBS and other supplements. SLR21, SLR22, SLR23, SLR24, SLR25, SLR26, KC-12 and 769-P cell lines ${ }^{46}$ were 
cultured in RPMI1640 supplemented with 10\% FBS and other supplements. Hep3B cells were cultured in MEM/EBSS (Thermo Scientific, Waltham, MA, USA) with $10 \%$ FBS and other supplements. When hypoxia treatment was needed, HEPES was added to the growth medium at final concentration of $25 \mathrm{~mm}$ and cells were placed in normoxia $\left(\mathrm{Nx}, 21 \% \mathrm{O}_{2}\right)$ or hypoxia $(\mathrm{Hx}, 1.5 \%$ $\mathrm{O}_{2}$ ) for $12-16 \mathrm{~h}$ for HIF target gene studies. RCC4, 786-O and SLR25 cells were authenticated by DNA profiling or 'fingerprinting' by the University of Colorado DNA Sequencing and Analysis Core and have been determined to be mycoplasma free.

Plasmid constructs and stable transfection

BAF180 CDNA amplified from Hep3B cells was inserted into the pLEX-MCS (Thermo Scientific) or pCW57.1 vector (Addgene \#41393, Cambridge, MA, USA; deposited by David Root laboratory). These lentiviral vectors were then transfected into 293T cells with other packaging plasmids to generate viruses that were used to obtain RCC4 or SLR25 cells expressing BAF180. The pcDNA3.1-hygro mouse HIF1A-Flag CDNA vector has been previously described. ${ }^{16}$ The vector linealized by Fspl was transfected into 786-O cells by Lipofectamine (Invitrogen, Waltham, MA, USA). The stably transfected cells were generated by hygromycin B selection.

shRNA-mediated knockdown of endogenous messenger RNAs pLKO.1 lentivirus expressing the non-targeting scrambled shRNA (SHC202), HIF1A shRNA (TRCN0000003810), HIF2A shRNA (TRCN0000003803) or PBRM1/BAF180 shRNAs (TRCN0000235892 or TRCN0000235890) were from Open Biosystems (Lafayette, CO, USA). Lentiviruses expressing shRNA were generated by transfection of the pLKO.1 shRNA construct with other viral protein expression vectors into 293T cells using TransIT (Mirus, Madison, WI, USA). The knockdown cells were generated by infection with the shRNA virus and puromycin selection.

CRISPR-Cas9-mediated editing of BAF180 gene in 786-O cells pCW-Cas9 (doxycycline-inducible Cas9 expression; \#5066) and pLX-sgRNA (lentiviral vector express AAVS1 sgRNA, \#50662) were purchased from Addgene. ${ }^{47}$ pLX-sgRNA was first modified by PCR mutagenesis by replacing the AAVS1 sgRNA with two $B s p M I$ recognition sites to generate a sgRNA cloning vector, pLX-sgRNA-BspMI. Oligos for the PBRM1/BAF180 target sequence were: sgRNA\#1 (5'-CACCGCTGACACTGCTGGAAGG-3', $5^{\prime}$-AAACCCCCTTCCAGCAGTGTCAG-3') and sgRNA\#2 (5'-CACCGTCATTAGG GCACCAAAG-3', 5'-AAACTCGCTTTGGTGCCCTAATG-3'). Each pair of target oligos was first annealed and then ligated into pLX-sgRNA-BspMI vector that had been cut with BspMI. To generate 786 cells with BAF180 knockout, first, 786-O/Tet-on Cas 9 cells were generated by transducing $786-\mathrm{O}$ cells with a pCW-Cas9 lentivirus. Subsequently, 786-O/Tet-on Cas9 cells were transduced with BAF180 sgRNA lentivirus and selected for 7 days with blasticidin $\mathrm{S} \mathrm{HCl}(12 \mu \mathrm{g} / \mathrm{ml})$ and $1 \mu \mathrm{g} / \mathrm{ml}$ doxycycline. Individual cells were then plated in 96-well plates. BAF180 expression in each subsequent clone was determined by western blotting, from which $~ 5 \%$ clones were determined to be BAF180 negative. Clones with loss of BAF180 protein expression were further confirmed by sequencing of the BAF180 genomic locus.

\section{Western blotting analysis}

Protein lysates were prepared with whole cell extraction buffer $(150 \mathrm{~mm}$ $\mathrm{NaCl}, 5 \mathrm{~mm}$ EDTA, $50 \mathrm{~mm}$ Tris pH 8.0, 0.1\% SDS, $1 \mathrm{~mm}$ PMSF and $1 \times$ Pierce protease inhibitor cocktail). Total protein concentration was determined using bicinchoninic acid assay reagents (Pierce, Waltham, MA, USA). For western blot analysis, $25 \mu \mathrm{g}$ of total protein was separated on an $8 \%$ SDSpolyacrylamide gel electrophoresis gel. The following primary antibodies were used: anti-HIF1a polyclonal (pAb) (NB100-134; Novus Biologicals, Littleton, CO, USA), anti-BAF180 pAb (NB100-79833; Novus Biologicals), anti-HIF2a mAb (D9E3, Cell Signaling Technology Inc., Danvers, MA, USA), anti-ARNT Ab (NB100-124, Novus Biologicals) and anti-actin pAb (SC-1616; Santa Cruz, Santa Cruz, CA, USA).

RNA isolation and reverse transcriptase-quantitative PCR analysis RNA was extracted from cells using the RNeasy Plus mini kit (Qiagen, Venlo, Netherlands) followed by reverse transcription using iScript Advanced cDNA Synthesis Kit (Bio-Rad, Hercules, CA, USA). Quantification of messenger RNA levels was performed by reverse transcription quantitative PCR (RT-qPCR) using iQ Universal SYBR Green Supermix (Bio-Rad) and
CFX384 Real Time System (Bio-rad). All primer sets for RT-qPCR were validated for their specificity and amplification efficiency (85\% to $110 \%)$ using melt curve analysis, RT-qPCR product sequencing and standard dilution analysis (Supplementary Table 1). The qPCR results were analyzed using the $\triangle \triangle C T$ method using $18 \mathrm{~S}$ ribosomal RNA and beta actin messenger RNA as reference genes and presented in relative to samples from control cells. At least three independent experiments were performed for all the results presented in this paper.

Detection of HIF1A gene deletions in ccRCC cell lines Genomic DNA was isolated from each ccRCC cell line using a QIAamp DNA mini kit (Qiagen). Twenty ng of genomic DNA was used in each qPCR reaction to quantify individual HIF1A exons using primer stets (Supplementary Table I) located in exons 2-15. Results of USF1 exon 11 and LMNA exon 7-intron 7 junctions were used as genomic DNA concentration controls. The DNA from HK2 cells was used as normal DNA content calibrator.

\section{Clonogenic survival assay}

Cells (250) were plated per well of a six-well plate, and cultured in 1\% FBS and $1 \times$ insulin-transferrin-selenium for 7-10 days. For Tet-on BAF180expressing cells, PBS or $1 \mu \mathrm{g} / \mathrm{ml}$ doxycycline were added to the medium. After 7-10 days, cells were washed with PBS once and stained with crystal violet $(50 \%$ glutaraldehyde and $0.5 \%(\mathrm{w} / \mathrm{v})$ crystal violet). Quantification was performed using MetaMorph software (Nashville, TN, USA) to measure the total area covered by colonies.

Immunohistochemistry analysis of BAF180 and HIF1a in CCRCC tumors

ccRCC Tissue Microarrays were obtained from US Biomax, Inc (cat. \#BC07014a). Immunohistochemical staining using HIF1a (Thermo Fisher Scientific, Waltham, MA, USA, H1alpha67) or BAF180 (Novus Biologicals; NB100-79833) antibodies was performed on two slides containing the same tumors according to standard immunohistochemistry protocols.

\section{Nucleosome scanning assay}

Hep3B cells expressing shRNA against BAF180 or BRG1, or non-targeting shRNA ( $60 \%$ confluency) were cultured under normoxic or HX conditions for 16 hours and nucleosomal DNA was isolated using the EZ Nucleosomal DNA prep kit (Zymo Research, Irvine, CA, USA). The primer sets (Supplementary Table 2) and the procedure for quantification of the CA9 promoter region were described previously. ${ }^{23}$

\section{Statistical analysis}

One-way analysis of variance followed by post hoc test (two-sample $t$-test with Bonferroni correction) was performed for statistical analysis unless otherwise stated. Error bars in figures indicate s.d. Asterisks indicate statistical significance as follows: ${ }^{*} P<0.01,{ }^{*} P<0.03$. Controls for statistical analysis are specified in each figure. All experiments were performed at least three separate times.

\section{CONFLICT OF INTEREST}

The authors declare no conflict of interest.

\section{ACKNOWLEDGEMENTS}

This work was partially supported by grants from 2014 Cancer League of Colorado Research Fund (C-JH), University of Colorado School of Dental Medicine Bridge Fund $(\mathrm{C}-\mathrm{JH})$ and the National Institute of Environmental Health Sciences (R01ES020868, B-HJ).

\section{REFERENCES}

1 Siegel RL, Miller KD, Jemal A. Cancer statistics, 2016. CA Cancer J Clin 2016; 66: 7-30.

2 Cairns P. Renal cell carcinoma. Cancer Biomark 2010; 9: 461-473.

3 Weiss $\mathrm{RH}$, Lin PY. Kidney cancer: identification of novel targets for therapy. Kidney Int 2006; 69: 224-232.

4 Kaelin WG Jr. The von Hippel-Lindau tumor suppressor protein and clear cell renal carcinoma. Clin Cancer Res 2007; 13: 680s-684ss. 
5 Jonasch E, Futreal PA, Davis IJ, Bailey ST, Kim WY, Brugarolas J et al. State of the science: an update on renal cell carcinoma. Mol Cancer Res 2012; 10: 859-880.

6 Porta C, Giglione P, Paglino C. Targeted therapy for renal cell carcinoma: focus on 2nd and 3rd line. Expert Opin Pharmacother 2016; 17: 1-13.

7 Varela I, Tarpey P, Raine K, Huang D, Ong CK, Stephens P et al. Exome sequencing identifies frequent mutation of the SWI/SNF complex gene PBRM1 in renal carcinoma. Nature 2011; 469: 539-542.

8 Duns G, Hofstra RM, Sietzema JG, Hollema H, van Duivenbode I, Kuik A et al. Targeted exome sequencing in clear cell renal cell carcinoma tumors suggests aberrant chromatin regulation as a crucial step in ccRCC development. Hum Mut 2012; 33: 1059-1062.

9 Gerlinger M, Rowan AJ, Horswell S, Larkin J, Endesfelder D, Gronroos E et al. Intratumor heterogeneity and branched evolution revealed by multiregion sequencing. N Engl J Med 2012; 366: 883-892.

10 Cancer Genome Atlas Research N. Comprehensive molecular characterization of clear cell renal cell carcinoma. Nature 2013; 499: 43-49.

11 Sato $Y$, Yoshizato T, Shiraishi Y, Maekawa S, Okuno Y, Kamura T et al. Integrated molecular analysis of clear-cell renal cell carcinoma. Nat Genet 2013; 45: 860-867.

12 Maxwell PH, Wiesener MS, Chang GW, Clifford SC, Vaux EC, Cockman ME et al. The tumour suppressor protein VHL targets hypoxia-inducible factors for oxygen-dependent proteolysis. Nature 1999; 399: 271-275.

13 Kaelin WG Jr. Cancer and altered metabolism: potential importance of hypoxia-inducible factor and 2-oxoglutarate-dependent dioxygenases. Cold Spring Harb Symp Quant Biol 2011; 76: 335-345.

14 Keith B, Johnson RS, Simon MC. HIF1alpha and HIF2alpha: sibling rivalry in hypoxic tumour growth and progression. Nat Rev Cancer 2012; 12: 9-22.

15 Semenza GL. Hypoxia-inducible factors in physiology and medicine. Cell 2012; 148: 399-408.

$16 \mathrm{Hu}$ CJ, Wang LY, Chodosh LA, Keith B, Simon MC. Differential roles of hypoxia-inducible factor 1alpha (HIF-1alpha) and HIF-2alpha in hypoxic gene regulation. Mol Cell Biol 2003; 23: 9361-9374.

17 Raval RR, Lau KW, Tran MG, Sowter HM, Mandriota SJ, Li JL et al. Contrasting properties of hypoxia-inducible factor 1 (HIF-1) and HIF-2 in von Hippel-Lindau-associated renal cell carcinoma. Mol Cell Biol 2005; 25: 5675-5686.

18 Schönenberger D, Harlander S, Rajski M, Jacobs RA, Lundby A-K, Adlesic M et al. Formation of renal cysts and tumors in Vhl/Trp53-deficient mice requires HIF1a and HIF2a. Cancer Res 2016; 76: 2025-2036.

19 Kondo K, Kim WY, Lechpammer M, Kaelin WG Jr. Inhibition of HIF2alpha is sufficient to suppress pVHL-defective tumor growth. PLoS Biol 2003; 1: E83.

20 Kondo K, Klco J, Nakamura E, Lechpammer M, Kaelin WG Jr. Inhibition of HIF is necessary for tumor suppression by the von Hippel-Lindau protein. Cancer Cell 2002; 1: 237-246.

21 Zimmer M, Doucette D, Siddiqui N, lliopoulos O. Inhibition of hypoxia-inducible factor is sufficient for growth suppression of $\mathrm{VHL}^{-/-}$tumors. Mol Cancer Res 2004; 2: 89-95

22 Gordan JD, Lal P, Dondeti VR, Letrero R, Parekh KN, Oquendo CE et al. HIF-alpha effects on c-Myc distinguish two subtypes of sporadic VHL-deficient clear cell renal carcinoma. Cancer Cell 2008; 14: 435-446.

23 Sena JA, Wang L, Hu CJ. BRG1 and BRM chromatin-remodeling complexes regulate the hypoxia response by acting as coactivators for a subset of hypoxia-inducible transcription factor target genes. Mol Cell Biol 2013; 33: 3849-3863.

24 Gordan JD, Bertout JA, Hu CJ, Diehl JA, Simon MC. HIF-2alpha promotes hypoxic cell proliferation by enhancing c-myc transcriptional activity. Cancer Cell 2007; 11: 335-347.

25 Pawlus MR, Wang L, Hu CJ. STAT3 and HIF1alpha cooperatively activate HIF target genes in MDA-MB-231 and RCC4 cells. Oncogene 2014; 33: 1670-1679.

26 Shen C, Beroukhim R, Schumacher SE, Zhou J, Chang M, Signoretti S et al. Genetic and functional studies implicate HIF1alpha as a $14 q$ kidney cancer suppressor gene. Cancer Disc 2011; 1: 222-235.

27 Shuib S, Wei W, Sur H, Morris MR, McMullan D, Rattenberry E et al. Copy number profiling in von Hippel-Lindau disease renal cell carcinoma. Genes Chromosomes Cancer 2011; 50: 479-488.

28 Chowdhury B, Porter EG, Stewart JC, Ferreira CR, Schipma MJ, Dykhuizen EC. PBRM1 regulates the expression of genes involved in metabolism and cell adhesion in renal clear cell carcinoma. PLoS ONE 2016; 11: e0153718.
29 Macher-Goeppinger S, Keith M, Tagscherer KE, Singer S, Winkler J, Hofmann TG et al. PBRM1 (BAF180) protein is functionally regulated by $\mathrm{p} 53$-induced protein degradation in renal cell carcinomas. J Pathol 2015; 237: 460-471.

30 Cairns BR. The logic of chromatin architecture and remodelling at promoters. Nature 2009; 461: 193-198.

31 Hargreaves DC, Crabtree GR. ATP-dependent chromatin remodeling: genetics, genomics and mechanisms. Cell Res 2011; 21: 396-420.

32 Trotter KW, Archer TK. The BRG1 transcriptional coregulator. Nucl Recept Signal 2008; 6: e004.

$33 \mathrm{Hu} \mathrm{CJ}$, Sataur A, Wang L, Chen H, Simon MC. The N-terminal transactivation domain confers target gene specificity of hypoxia-inducible factors HIF-1alpha and HIF-2alpha. Mol Biol Cell 2007; 18: 4528-4542.

34 Warnecke C, Zaborowska Z, Kurreck J, Erdmann VA, Frei U, Wiesener M et al. Differentiating the functional role of hypoxia-inducible factor (HIF)-1alpha and HIF-2alpha (EPAS-1) by the use of RNA interference: erythropoietin is a HIF-2alpha target gene in Hep3B and Kelly cells. FASEB $J$ 2004; 18: 1462-1464.

35 Pawlus MR, Wang L, Ware K, Hu CJ. Upstream stimulatory factor 2 and hypoxiainducible factor 2alpha (HIF2alpha) cooperatively activate HIF2 target genes during hypoxia. Mol Cell Biol 2012; 32: 4595-4610.

36 Biswas S, Troy H, Leek R, Chung YL, Li JL, Raval RR et al. Effects of HIF-1alpha and HIF2alpha on Growth and Metabolism of Clear-Cell Renal Cell Carcinoma 786-0 Xenografts. J Oncol 2010; 2010: 757908 .

$37 \mathrm{Wu}$ JN, Roberts CWM. ARID1A mutations in cancer: another epigenetic tumor suppressor? Cancer discovery 2013; 3: 35-43.

38 Pena-Llopis S, Vega-Rubin-de-Celis S, Liao A, Leng N, Pavia-Jimenez A, Wang S et al. BAP1 loss defines a new class of renal cell carcinoma. Nat Genet 2012; 44: 751-759.

39 Nakazato H, Takeshima H, Kishino T, Kubo E, Hattori N, Nakajima $\mathrm{T}$ et al. Early-stage induction of SWI/SNF mutations during esophageal squamous cell carcinogenesis. PLoS ONE 2016; 11: e0147372.

40 Thompson M. Polybromo-1: the chromatin targeting subunit of the PBAF complex. Biochimie 2009; 91: 309-319.

41 Jiao Y, Pawlik TM, Anders RA, Selaru FM, Streppel MM, Lucas DJ et al. Exome sequencing identifies frequent inactivating mutations in BAP1, ARID1A and PBRM1 in intrahepatic cholangiocarcinomas. Nat Genet 2013; 45: 1470-1473.

42 Huang L, Peng Y, Zhong G, Xie W, Dong W, Wang B et al. PBRM1 suppresses bladder cancer by cyclin B1 induced cell cycle arrest. Oncotarget 2015; 6: 16366-16378.

43 Pawlowski R, Muhl SM, Sulser T, Krek W, Moch H, Schraml P. Loss of PBRM1 expression is associated with renal cell carcinoma progression. Int J Cancer 2013; 132: E11-E17.

44 Xia W, Nagase S, Montia AG, Kalachikov SM, Keniry M, Su T et al. BAF180 is a critical regulator of p21 induction and a tumor suppressor mutated in breast cancer. Cancer Res 2008; 68: 1667-1674.

45 Burrows AE, Smogorzewska A, Elledge SJ. Polybromo-associated BRG1-associated factor components BRD7 and BAF180 are critical regulators of p53 required for induction of replicative senescence. Proc Natl Acad Sci USA 2010; 107: $14280-14285$.

46 Tatsumi T, Herrem CJ, Olson WC, Finke JH, Bukowski RM, Kinch MS et al. Disease stage variation in $\mathrm{CD}^{+}$and $\mathrm{CD}^{+}{ }^{+}$-cell reactivity to the receptor tyrosine kinase EphA2 in patients with renal cell carcinoma. Cancer Res 2003; 63: 4481-4489.

47 Wang T, Wei JJ, Sabatini DM, Lander ES. Genetic screens in human cells using the CRISPR-Cas9 system. Science 2014; 343: 80-84.

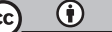

Oncogenesis is an open-access journal published by Nature Publishing Group. This work is licensed under a Creative Commons Attribution 4.0 International License. The images or other third party material in this article are included in the article's Creative Commons license, unless indicated otherwise in the credit line; if the material is not included under the Creative Commons license, users will need to obtain permission from the license holder to reproduce the material. To view a copy of this license, visit http://creativecommons.org/licenses/by/4.0/

(c) The Author(s) 2017

Supplementary Information accompanies this paper on the Oncogenesis website (http://www.nature.com/oncsis). 